\title{
ELETROMIOGRAFIA APLICADA AOS MÚSCULOS DA MASTIGAÇÃOO
}

\author{
ELECTROMYOGRAPHY APPLIED TO CHEWING MUSCLES
}

\author{
Julana Malta', Gabriel Denser Campolongo², Tarley Eloy Pessoa de Barros ${ }^{3}$, Reginaldo Perilo de Olineira ${ }^{4}$
}

\section{RESUMO}

A eletromiografia tem se mostrado um método eficaz no auxilio de diagnósticos e tratamentos de desordens musculares. Na odontologia sua principal aplicação é no tratamento das Disfunções Temporomandibulares. Os profissionais de odontologia não utilizam esta técnica por desconhecerem seus benefícios ou mesmo não terem conhecimento sobre o assunto.

Um dos principais objetivos do tratamento utilizando eletromiografia é mostrar ao paciente o estado atual do músculo analisado e sua evolução durante o tratamento, até chegar a uma função satisfatória.

Descritores: Eletromiografia; Síndrome da disfunção da articulação temporomandibular; Articulação temporomandibular.

\section{INTRODUÇÃO}

A eletromiografia tem sido amplamente utilizada no diagnóstico clínico há mais de 40 anos. Originalmente foi utilizada pela neurofisiologia, o termo "eletromiografia" foi então usado como referência aos métodos empregados para registrar os potenciais de ação das fibras musculares de pacientes saudáveis e patológicos ${ }^{(1)}$.

O monitoramento da atividade dos músculos através da eletromiografia (EMG) é uma forma insubstituível para se verificar as condições fisiológicas do sistema estomatognático ${ }^{(1)}$.

A eletromiografia de superfície é atualmente uma parte da avaliação que quantifica a função dos músculos da mastigação dos pacientes na Odontologia. Certamente, uma aproximação dos valores reais do músculo analisado poderia ser valiosa no diagnóstico das alterações do sistema estomatognático e na avaliação dos efeitos terapêuticos ${ }^{(2,3,4)}$. Pode ser usada para entender mais profundamente as diversas patologias, como por exemplo as desordens temporomandibulares ${ }^{(3)}$.

Apresenta-se como um método seguro, fácil, e não invasivo que permite a quantificação objetiva da energia do músculo estudado(5).

A eletromiografia tem sido de grande importância para o conhecimento da fisiologia muscular in vivo, no diagnóstico diferencial e monitoramento das possíveis desordens ${ }^{(4)}$.

Conhecendo-se o que é normal, pode-se fazer um diagnóstico diferencial das condições que se apresentam anormais ${ }^{(4)}$. Procurou-se verificar quais as áreas de atuação da eletromiografia na Odontologia, se seu uso se restringiria apenas as Disfunções Temporamandibulares ou teria utili-

\section{SUMMARY}

The electromyography has if shown an effective method in the aid of diagnoses and treatments of muscular disorders. In dentistry your main application it is in Dysfunction Temporomandibular treatment. The dentistry professionals don't use this technique for they ignore their benefits or even they have not knowledge on the subject.

One of the main objectives of the treatment using electromyography is to show to the patient the current state of the analyzed muscle and your evolution during the treatment, until arriving to a satisfactory function.

Keywords: Electromyography; Temporomandibular joint dysfunction syndrome; Temporomandibular joint.

zação em áreas como a Cirurgia e Traumatologia Bucomaxilofacial.

\section{REVISÃO DE LITERATURA}

Atualmente a eletromiografia é empregada na avaliação do alcance da doença neuromuscular ou do traumatismo, e como um instrumento cinesiológico para estudo da função muscular. Como procedimento de avaliação, a eletromiografia clínica envolve a detecção e registro dos potenciais elétricos das fibras musculares esqueléticas ${ }^{(6)}$. A eletromiografia (EMG) é o registro elétrico da atividade de um músculo(5,7,8). Monitorando com sensores localizados na pele, sobre os músculos apropriados, onde são processados e reproduzidos na tela de um microcomputador. É utilizada no campo da Oclusão basicamente para avaliação do padrão de atividade do músculo mastigatório em função, bem como da posição postural de repouso da mandíbula ${ }^{(7)}$. Pode ser um método adicional e útil na avaliação de movimentos mastigatórios em Desordens Temporomandibulares ${ }^{(9)}$.

O estudo clínico eletrofísico consiste na observação, análise e interpretação da atividade bioelétrica e da integridade funcional do sistema neuromuscular em resposta à ativação ou estimulação elétrica. Para um melhor entendimento desses procedimentos serão apresentados alguns conceitos eletrofisiológicos básicos envolvidos no sistema neuromuscular ${ }^{(10)}$.

A contração muscular e a produção de forças são provocadas pela mudança relativa de várias moléculas ou filamentos no interior do músculo. O deslizamento dos filamentos é provocado por um fenômeno elétrico conhecido como potencial de ação(8).

Trabalho realizado na Faculdade de Odontologia da Universidade Bandeirante de São Paulo e no Instituto de Ortopedia e Traumatologia do HC/FMUSP

Endereço para Correspondência: Tarley Eloy Pessoa de Barros - Rua Guarará, 316 - e-mail:unidor@unidor.com.br

1. Aluna do Curso de Especialização em Cirurgia e Traumatologia Bucomaxilofacial da Universidade Bandeirante de São Paulo

2. Pós-Graduando, nível mestrado, no Departamento de Ortopedia e Traumatologia da Faculdade de Medicina da USP.

3. Pós-Graduando, nível Doutorado, no Departamento de Ortopedia e Traumatologia da Faculdade de Medicina da USP.

4. Médico Assistente do Instituto de Ortopedia e Traumatologia da Faculdade de Medicina da USP.

Trabalho recebido em: 17/11/05 aprovado em 31/01/05 
Potencial de ação é a despolarização do músculo que ocorre quando o axônio conduz um impulso para todas as suas fibras musculares. Esse potencial de ação é graficamente registrado pelo eletromiograma ${ }^{(6,8,10)}$.

Dessa forma, um potencial de ação transmitido ao longo de uma fibra nervosa é denominado impulso nervoso, enquanto detectável através de eletrodos sobre a pele ou por eletrodos de agulha inseridos no músculo sendo que, em ambos os casos, o distúrbio elétrico registrado é a soma dos potenciais produzidos por todas as fibras musculares $\operatorname{ativadas}^{(10)}$.

$\mathrm{Na}$ eletromiografia, pode-se registrar os potenciais de ação que ocorrem através da ativação voluntária do músculo ou através da resposta deste, frente a uma estimulação elétrica ${ }^{(10)}$. A eletromiografia não traduz uma medida de força, uma vez que se sabe que a eletromiografia e a força dissociamse quando um músculo está fadigado ou em contrações não isométricas ${ }^{(10)}$.

Os eletrodos que capturam os potenciais elétricos do músculo em contração podem ser de dois tipos:

Eletrodos de Superfície: são pequenos discos metálicos, mais comumente feitos de prata-cloreto de prata, colocados sobre a pele. São muito mais convenientes para os clínicos, mais aceitáveis pelos pacientes e produzem menos movimentos., método não invasivo e de fácil execução. Usado para músculos superficiais. Faz-se necessária a preparação da pele, que pode ser a lavagem ou fricção com álcool, para remoção das células epiteliais mortas. Aplica-se gel de eletrodo, para que seja facilitada a condução dos potenciais elétricos. O eletrodo de ser firmemente aderido à pele, com fitas adesivas, para evitar interferências ao serem realizados os movimentos ${ }^{(6,7,8,10,11)}$.

Eletrodos de profundidade: os eletrodos são colocados no interior do músculo, utilizando-se dois filamentos de arame de pequeno calibre, revestidos, que são introduzidos através de uma agulha hipodérmica. Pouco utilizado por ser um método invasivo ${ }^{(6,8,10,12)}$.

Os eletrodos ainda podem ser classificados como:

Monopolar: um eletrodo é colocado sobre o feixe muscular de interesse e o outro eletrodo é colocado num ponto não afetado pela atividade do feixe muscular de interesse, mede-se então a diferença de potencial entre esse dois pontos ${ }^{(8)}$.

Bipolar: dois eletrodos sobre a região que se deseja estudar e o terceiro eletrodo chamado terra é colocado num local não afetado pela atividade da região de interesse. Mede-se agora a diferença de potencial elétrico entre os dois eletrodos que estão sobre a região de interesse, tomando-se como referência o eletrodo terra ${ }^{(3,7,8)}$.

\section{DISCUSSÃO}

Os autores relatam que a eletromiografia tem grande aplicação nas Disfunções Temporomandibulares, onde se verifica sua atuação na análise dos músculos da mastigação. Observou-se unanimidade na utilização de eletrodos de superfícies por ser de fácil execução, não invasivo e que não traz nenhum desconforto ao paciente.

Todos os autores afirmam a possível utilização da eletromiografia em outras áreas da Odontologia, porém discutem apenas sobre sua utilização nas Disfunções Temporomandibulares.

A eletromiografia poderia ser utilizada em áreas como a Ortodontia como meio de diagnosticar uma possível alteração muscular antes do inicio do tratamento e verificando uma possível reorganização muscular pós-tratamento, observando se esse músculo conseguiu com sucesso retornar as sua atividades fisiológicas normais.

Poderia ainda ser utilizada na Cirurgia e Traumatologia Bucomaxilofacial também verificando as funções musculares, alteradas antes da cirurgia, observando suas adaptações pós-cirurgia e sua reorganização até chegar a uma função satisfatória.

Observa-se ainda, através da eletromiografia as alterações musculares de pacientes que necessitem de reabilitação protética podendo-se afirmar que a necessidade de reabilitação interfere na harmonia muscular interferindo assim na função da Articulação Temporomandibular.

\section{CONCLUSÕES}

O exame eletromiográfico mostrou-se de grande eficiência no diagnóstico auxiliar e preventivo das disfunções que envolvam a musculatura d sistema estomatognático.

Recursos do eletrodiagnóstico ainda encontram-se distantes de uma realidade profissional concreta, devido à falta de conhecimento da técnica acompanhado ao alto custo dos aparelhos.

Com os resultados obtidos através da eletromiografia podese comprovar de forma exata, e mostrar ao paciente, que tratamentos ortodônticos, cirurgias e reabilitação não são indicados apenas pelo resultado estético final e sim pelo resultado funcional que apresenta em relação à oclusão, posicionamento da articulação e função muscular que é comprovada pela eletromiografia.

\section{REFERÊNCIAS BIBLIOGRÁFICAS}

1. Altruda Filho L, Larossa Pr, Tessler M. Avaliação eletromiográfica de pacientes portador de maloclusão de classe II, DIV. $2^{\circ}$ de Angle - Caso Clínico Pré e Pós Tratamento. Disponível em URL: http://www.papaizassociados.com.br/PapaizNews/trabalho\%(BASSANTA)0eletromio.htm (Último acesso em 27/06/2005)

2. Bevilaqua-Grosso D, Monteiro-Pedro V, Jesus Gruirro RR. A physiotherapeutic approach to craniomandibular disorders: a case report. J Oral Rehabil. 2002; 29:268-73.

3. Ferrario VF, Sforza C, Tartaglia GM, Dellavia C. immediate effect of a stabilization splint on masticatory muscle activity in temporomandibular disorder patients. J Oral Rehabil. 2002; 29:810-15.

4. Landulpho AB, Silva WAB, Silva FA. Eletromiografia e eletrosonografia no monitoramento da desordens temporomandibulares - relato de caso clínico. Rev Paul Odontol. 2003; 25:4-8.

5. Goldstein LB. The use of surface electromyography in objective measurement of the muscle function in facial pain / temporomandibular dysfunction patients. Funct Orthod. 2000; 17:26-9.

6. O' Sullivan SB, Schmitz TJ. Eletromiografia e testes de velocidade de condução nervosa. In: Fisioterapia - Avaliação e tratamento. 2a. ed. São Paulo: Manole;1993. p. 183-218.

7. BassantaAD, Sproesser JG, Paiva G. Estimulação neural transcutânea ("TENS"): sua aplicação na disfunções temporomandibulares. Rev Odontol Univ São Paulo. 1997;11:109-16.

8. Rodriguez-Añez CR. A Eletromiografia na análise da postura. Disponível em URL http://winston.alhosting.com.br/emg_e postura.htm (Último acesso em: 06/09/2004).

9. Sato S, Nasu F, Motegi K. Analysis of post-treatment electromyographs im patients with non-reducing disc displacement of the temporomandibular. J Oral Rehabil. 2002; 9:1126-30.

10. Torriani C, Cyrillo FN. Biofeedback: conceitos básicos e aplicabilidade clínica. Rev Fisioter UniFMU. 2003; 1:11-8.

11. Turcio KHL, Garcia AR, Derogis AR, Zuim PRJ. Avaliação eletromiográfica e eletrovibratógráfica antes e após o tratamento da desordem temporomandibular. Pós Graduação em Revista - Odontologia. 2002; 5:36-43.

12. Laat $A D$, Komiyama $O$. Qual a utilidade da eletromiografia no diagnóstico de dor muscular na mandíbula ou disfunções temporomandibulares? Rev Int Ortop Func. 2004; 1:71-84. 